\title{
Fragebogen hilft zwischen Durchbruchschmerz und mangelnder Dauertherapie differenzieren
}

Häufig kommt es bei Tumorschmerzpatienten zu vorübergehenden Schmerzexazerbationen. Ob es sich dabei im Einzelfall um Durchbruchschmerzen oder eine insuffiziente medikamentöse Dauertherapie handelt, ist für die Patienten oftmals schwer zu unterscheiden. Hilfe bei der richtigen Einschätzung bietet der „DGS Praxisfragebogen zur Therapiesicherheit bei tumorbedingten Durchbruchschmerzen“.

\section{Durchbruchschmerzen besser identifizieren}

In Zusammenhang mit der neuen PraxisLeitlinie zur Therapie bei Durchbruchschmerzen entwickelte die Deutsche Gesellschaft für Schmerztherapie (DGS) den Praxisfragebogen. Er soll dazu beitragen, Durchbruchschmerzen besser zu erkennen und Hilfestellung bei differenzialtherapeutischen Überlegungen geben, erläuterte PD Dr. Michael Überall, Nürnberg.

20 Fragen zu verschiedenen Aspekten von Dauerschmerzen und Schmerzattacken können die Patienten selbst beantworten. Zudem enthält der Fragebogen einen Vordruck für den Arzt, auf dem er die Informationen des Patienten zusammenführt. So wird schnell deutlich, ob man aufgrund der Schmerzattacken eventuell die Dauertherapie optimieren oder die Bedarfsmedikation angepassen muss. Wenn Schmerzexazerbationen regelmäßig zum Ende des Dosierungsintervalls der Basismedikation (end of dose failure) auftreten, ist eine Dosisanpassung erforderlich. Bei Durchbruchschmerzen, spontan auftretenden Schmerzspitzen, können sehr schnell freisetzende Opioide (nasales oder oral- transmukosales Fentanyl) oder schnell freisetzende Opioide wie zum Beispiel nicht retardiertes orales Oxycodon, Hydromorphon die Bedarfsmedikation der ersten Wahl sein. Bei nicht vorhersehbaren Durchbruchschmerzen, ausgelöst etwa durch Husten, Niesen oder Defäkation, sind von Patienten selbst anwendbaren schnell freisetzenden Fentanylpräparate erste Wahl.

\section{Therapie individuell abstimmen}

Zudem können diese Präparate auch differenziert eingesetzt werden: Bei Patienten mit Dysphagie, Xerostomie oder Mukositis sind nasale Präparate von Vorteil. Bei Epistaxis oder nasalen Befunden sind dagegen oral-transmukosal anwendbare Substanzen eine Option. Ist das Auftreten von Durchbruchschmerzen vorhersehbar, etwa wenn sie immer morgens beim Aufstehen oder bei physiotherapeutischen Übungen einsetzen, können die Patienten die Schmerzen durch die rechtzeitige Einnahme von schnell wirksamem oralem Morphin, Oxycodon oder Hydromorphon verhindern. Diese Medikamente sind nicht mehr geeignet, wenn der Durchbruchschmerz bereits eingesetzt hat, da ihre Wirkung aufgrund der Magenpassage zu spät einsetzt und dann auch unnötigerweise über Stunden andauert. Der Praxisfragebogen wird auf der Homepage der DGS (www.dgschmerztherapie. de) als Download zur Verfügung gestellt. Ulrike Maronde

\section{Oft nicht erkannt: Schmerz bei Demenz}

Bei Demenzkranken können Verhaltensstörungen auch durch Schmerzen verstärkt werden. Eine adäquate Schmerztherapie bessert dann auch die Verhaltensprobleme. Aber Schmerzen werden bei Patienten mit fortgeschrittener Demenz häufig nicht ausreichend erkannt und erfasst - infolgedessen unterbleibt eine adäquate Schmerztherapie. „Patienten ohne Demenz erhalten dreimal so viele
Schmerzmittel wie Patienten mit Demenz", so Prof. Bettina Husebø, Bergen, Norwegen.

Schmerzen bei Demenzkranken zu erkennen, die keine Auskunft mehr geben können, bleibt eine Herausforderung. Hier sind beobachtungsbasierte Skalen wie BESD (BEurteilung von Schmerzen bei Demenz), ECPA (Echelle Comportemental de la douleur pour Personnes 